\title{
Robotic Hair Transplantation Surgery
}

\author{
Dr MS Khondoker ${ }^{\mathrm{a}}$, Dr R Awwal ${ }^{\mathrm{b}}$, Dr KMS Islam , Dr RE Rabbi ${ }^{\mathrm{d}}$
}

\section{Summary:}

Background: Hair transplantation is one of the most evolving procedures in aesthetic surgery accompanied by regular improvement in technique. The recent advancement in technology and the concept of using follicular unit grafts have made this procedure reach a new height. A precise appreciation of anatomy has allowed the use of follicular unit graft. With better methods of harvesting and implantation, hair transplantation results represent a blend of art and science. Materials \& method: 60 patients underwent hair transplant surgery from July/2008 to June/2009. Age limit was 18-58 years. Patients were evaluated and prepared in standard way. Operations were done by Robotic follicular unit graft by a specially designed machine. Procedures were performed under nerve block and local infiltration. Grafting sessions lasted for 5-6 hours, during which 1500-2000 FUGs were transplanted. Patients were discharged on the same day. Results: Minimum complications were observed. Most of the patients were satisfied except a few. Some patients needed second sitting surgery. Conclusion: With better methods of harvesting and implantation, Hair transplantation, if done judiciously, is a very rewarding procedure, both for the surgeon and the patient.

\section{Introduction :}

Hair has no vital function in humans, yet its psychological functions are extremely important. It imparts a youthful and attractive look to both males and females. Various studies have revealed that the person with a regular quantity of hair was rated as most attractive, virile, strong, active, and sharp in either sex. ${ }^{1}$.The negative image of pattern baldness is the underlying factor for the multi-million dollar alopecia treatment industry.

The total number of hair follicles for an adult human is estimated at 5 million with 1 million on the head of which 100,000 alone cover the scalp ${ }^{1-2}$. The basic hair follicle structure remains essentially the same throughout the range of mammalian species with modifications for specialized functions ${ }^{2}$.

a. Dr. Md. Sazzad Kondoker, MS(G. Surgery), FCPS (Surgery), MS(Plastic Surgery), Associate Professor, Department of Plastic Surgery, Dhaka Medical College and Hospital

b. Dr. Rayhana Awwal, Assistant Professor, FCPS,FRCS(Edin), MS(Plastic) Department of Plastic Surgery, Dhaka Medical College and Hospital

c. Dr K M Sadeq Islam, Student, MS(Plastic surgery), 3rd part, Dhaka Medical College\& Hospital, Dhaka.

d. Dr Reza e Rabbi Student, MS(Plastic surgery), 2nd part, Dhaka Medical College\& Hospital, Dhaka.

Address of correspondence: Dr Md Sazzad Khondoker, Associate Professor, Department of Plastic Surgery, Dhaka Medical College \& Hospital.

Tell: 88-02-9128658, Mobile:O1713063263

e-mail: skhondoker@yahoo.com.
The Human hair cycle is as follows ${ }^{3-6}$;

Phase I : anagen, active growth phase.

Phase II : catagen, a period of controlled regression of the hair follicle

Phase III : telogen, when the follicle is in a so-called resting state.

\section{Etiological factors in alopecia :}

Endocrine abnormalities, Genetic factors, Systemic illness, Drugs - anticancer drugs and toxic agents, Diet, Psychological abnormalities, Trauma, Infections Structural hair defects, Ionizing radiation, X-ray and gamma rays and others.

\section{Androgenic Alopecia (AGA)}

Most cases of hair loss are due to androgenic alopecia (AGA). ${ }^{2}$ Fifty percent of men by age 50 years and $40 \%$ of women by menopause have some degree of AGA. Hair loss is gradual, with miniaturization of genetically programmed hair follicles. Uptake, metabolism, and conversion of testosterone to dihydrotestosterone by 5 -alpha-reductase is increased in balding hair follicles. ${ }^{3-5}$ AGA appears different in men as compared with women. In men with AGA, hair loss occurs in the fronto-temporal regions and on the vertex of the scalp, depending on severity. ${ }^{6,7}$ In female AGA patients, it is more diffuse and located centroparietally. ${ }^{8}$ The frontal hairline is usually intact in women.

\section{Management:}

Treatment options are medical, surgical \& others. 


\section{Medical Treatment :}

A)Topical minoxidil - The only proven medications that are indicated for AGA.

B) Anti androgen therapy - CPA (cyproterone acetate) in combination with ethinyl-estradiol. Where CPA is not available, the aldosterone antagonist spironolactone has been given.

C) Oral finasteride - Finasteride in not indicated in women.

\section{Surgical procedures for hair restoration :}

A) Scalp reduction

B) Rotation or advancement transposition flaps

C) Scalp expansion by tissue expander \& reconstruction.

D) Hair transplantation

\section{Other Baldness Solution:}

A) Wigs-Wigs made from artificial or real hair, kept in place by adhesives, are becoming more naturallooking

B) Hair weaving-The procedure is not surgical but merely cosmetic in that it attaches extensions to existing hairs.

\section{Cosmetic Remedies :}

To reduce the visual effect of thinning hair, can always resort to camouflage.

\section{Newer concepts :}

A) Mesotherapy - Local infiltration of micronutrients

B) Hair Regrowth LASER : 20 - 24 sessions needed.

\section{Hair transplantation}

Hair transplantation is the only truly permanent solution to baldness.

Transplantation of permanent hairs from the back and sides of the scalp to balding areas in the front is a successful procedure. Restoration is possible because the hair follicles on the sides and back of the scalp are insensitive to the hormones that cause androgenic alopecia, so there is less chance of fallout. During surgical hair transplantation, hair follicles are redistributed in bald areas, where they grow hairs that continue to grow for the rest of the individual's life.

\section{Indication for hair transplantation}

Loss of frontal Hair line, recession at the temple area. more extensive hair loss on the top and crown area, adding density to thinned out area of scalp, correcting a poorly transplanted area, correcting less hair on eyebrows or on other body parts like beard, moustache, chest, eyelash etc. , scars due to injury, burns etc. in the scalp, beard, eyebrow.

\section{Different types of hair transplantation}

There are basically 2 method -

A) Strip method

B) Follicular unit extraction (stitch less method) FUE

\section{Considering the size of the graft -}

a) Macrograft (cylidrical or elliptical) : 1/8th inch size, 12-18 hairs per graft.

b) Minigraft : 3-4 hairs / graft

c) Micrograft : 1-2 hair / graft

\section{Materials and Methods}

60 Patients underwent hair transplantation surgery from July/2006 to June/2008 at a private aesthetic surgery centre in Dhaka. 55 patients were male and 5 patients were female. The age range was 18 to 58 years. All cases were suffering from Androgenic alopecia.

Pre-operatively all patients were evaluated routinely by

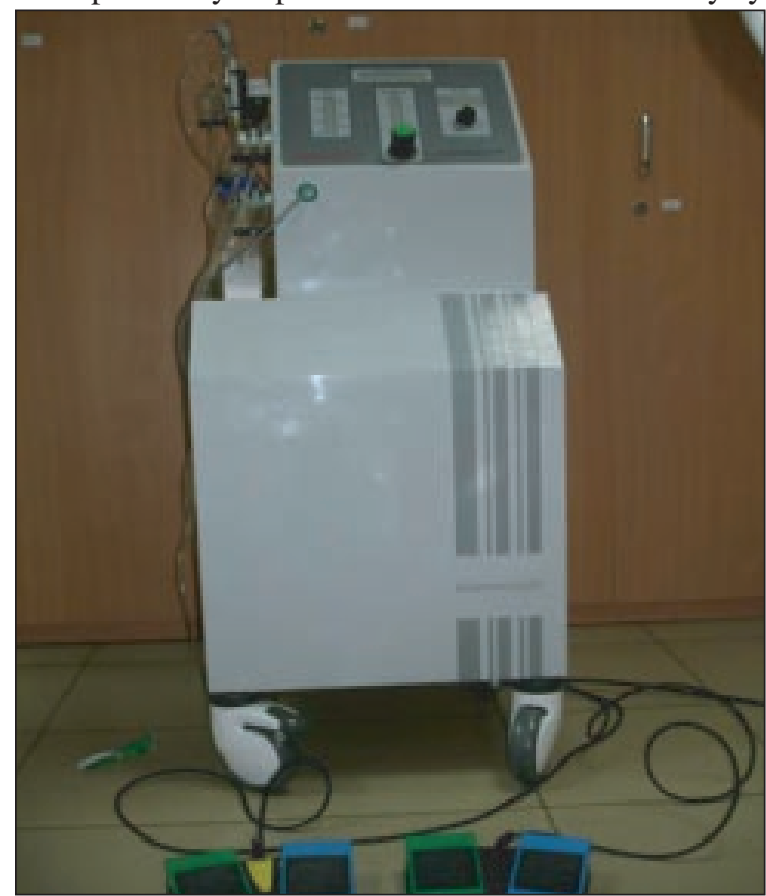

Figure 1: Hair transplantation machine 
proper history, clinical examination and investigation. Counseling was done routinely to explain the procedure $\&$ outcome.

Aspirin \& other blood thinning agents were prohibited

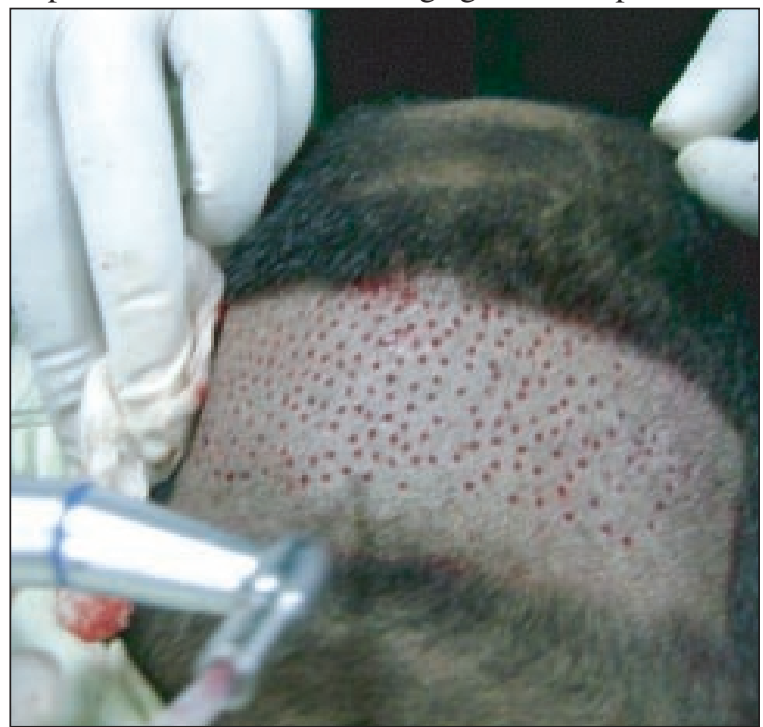

Figure 2: Follicle extraction

for $72 \mathrm{hrs}$ preoperatively. Patients were advised to avoid tea, coffee \& smoking at least 24 hours prior to the operation and advised to wash hair thoroughly

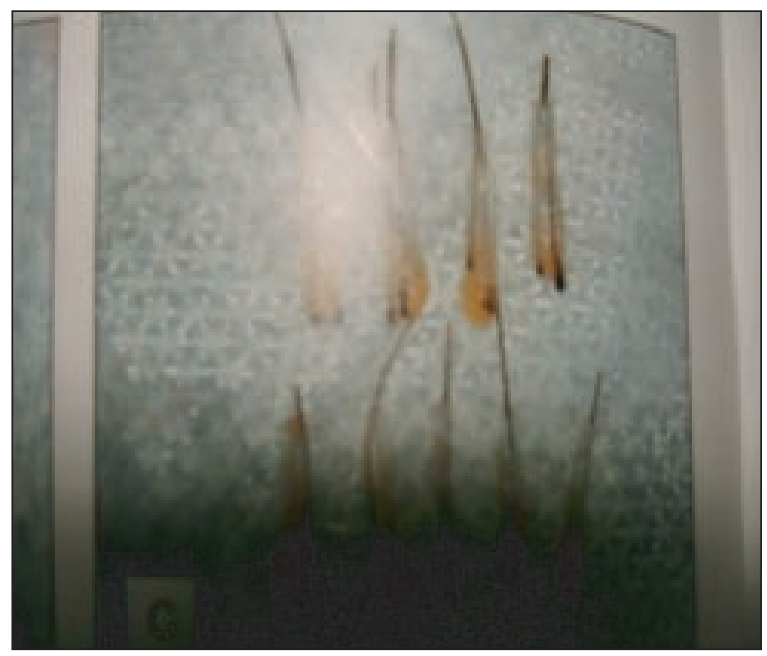

Figure 3: Preparation of the follicles

using regular shampoo. Donor area was prepared by hair trimming (fig:2).

Nerve block and local infiltration of anesthetic agent mixed with adrenaline was used in all cases.Hair Transplantation was done by a specific machine where graft i.e. follicular units were picked up or extracted by

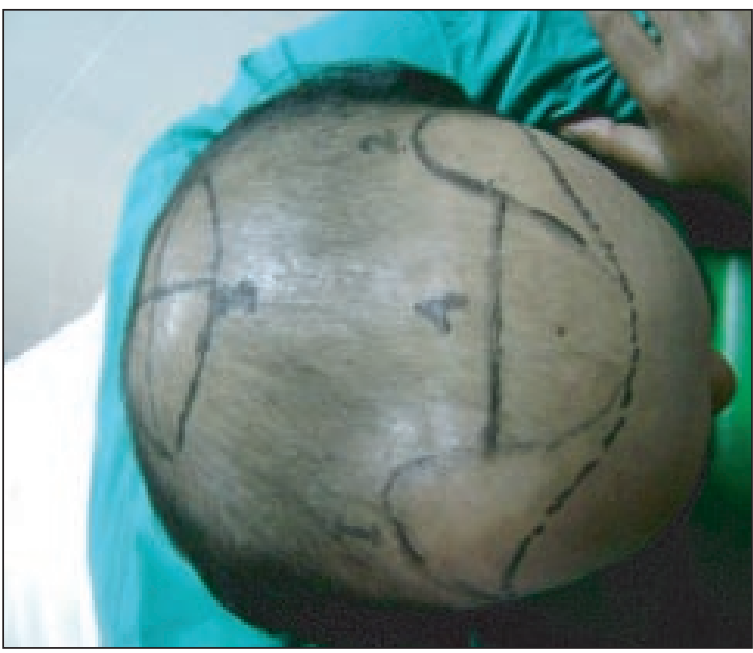

Figure 4: Planning in recipient area

high speed revolving sharp ended trocher with inflow suction pressure from donor area and preserved in a container in the machine (fig:2-4). Follicular units were washed. Injured follicles were removed (fig: 3 ). Following planning and designing the recipient bed was prepared by making small nick with knocker blade (fig: 4). Follicular unit were inserted by picking up by a nozzle with negative pressure and inserted in to the recipient whole by positive pressure (fig: 5). Total procedure was regulated by paddle switch.

At the end of the transplant, light non adhesive dressing

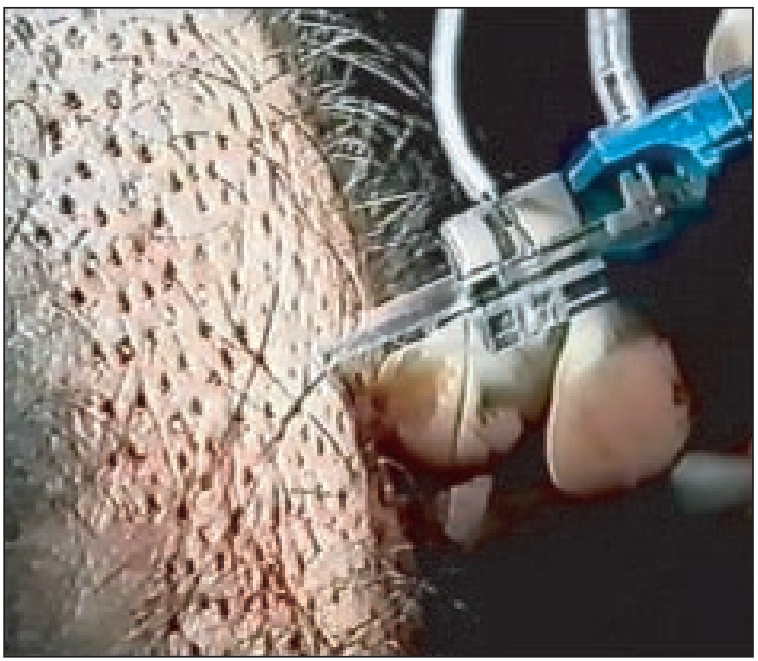

Figure 5: Follicular unit insertion

was applied to the scalp. Per operative antibiotic \& post operative analgesics were given and patients were allowed to leave hospital as day case with the advice to remove the dressing after 24 hours and apply topical 
antibiotic for 7 days. They were also advised to take rest for 3 days and resume normal work after 5 days.

Patients were followed up for 2 years.

\section{Observations and Results}

About 56 patients were followed up for 2 years. Four patients were lost from the follow up schedule in early post operative period. Most of the patients were satisfied at different levels. The levels of satisfaction were considered as excellent when take up was more than $90 \%$, good when take up was in between $90 \%$ to $70 \%$, poor when take-up was less than $70 \%$ upto $40 \%$ $\&$ failed where it was less than $40 \%$.

Complete failure observed in two cases. Exaggerated postoperative pain and paresthesia were observed in

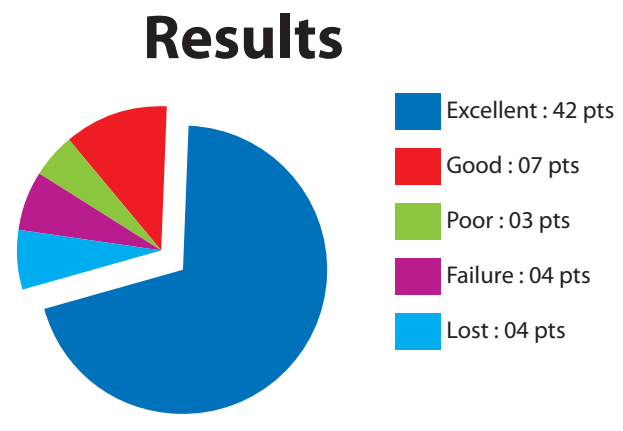

8 cases. Postoperative infection and hypertrophic scar were found in 4 cases. Postoperative psychiatric problems were observed in 5 patients

\section{Discussion}

Previously in Bangladesh hair transplant surgery used to be done by strip method. Hair transplantation surgery has improved in leaps and bounds over the past decade. The days of the "plugs and corn rows" are gone and the age of single hair, micro, and minigrafting has arrived. ${ }^{4,6}$

In this series 60 patients were treated over two years. Among which 42 patient exhibited excellent result, 7 patient exhibited good result and 3 patients showed poor result. These results are compatible with standard studies. ${ }^{9}$ Problem is with the number of patients leaving the study early. Rate of Post operative complications such as pain, Paresthesia, keloid \& hypertrophic scar formation \& misplacement of hairline was initially higher but with time the rate became compatible with standard studies. ${ }^{7,8}$ The overall level of satisfaction in part of patient is mostly good.
Some dissatisfactions were due to thin density, maldirected hair and misplacement of anterior hairline. The main causes of less satisfactions were, high expectation, poor counseling and inadequate post operative care. Complete failure observed in two cases where it may be due to poor patient selection and some technical failure.

\section{Conclusion}

Robotic follicular hair transplantation is an advanced, safe technology with non stitch \& non scalpel method

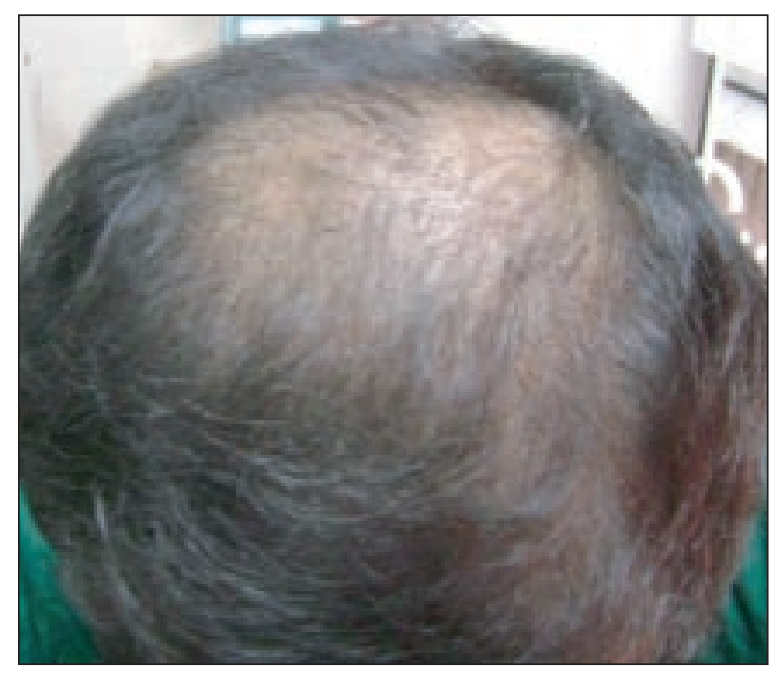

Figure 6: Before transplantation

and transplanted hair looks more natural. Single hair-grafts have the finest and softest appearance. Although they do not provide much density, they do provide the critical soft hairline. It is critically important to get it right at the first time and thus

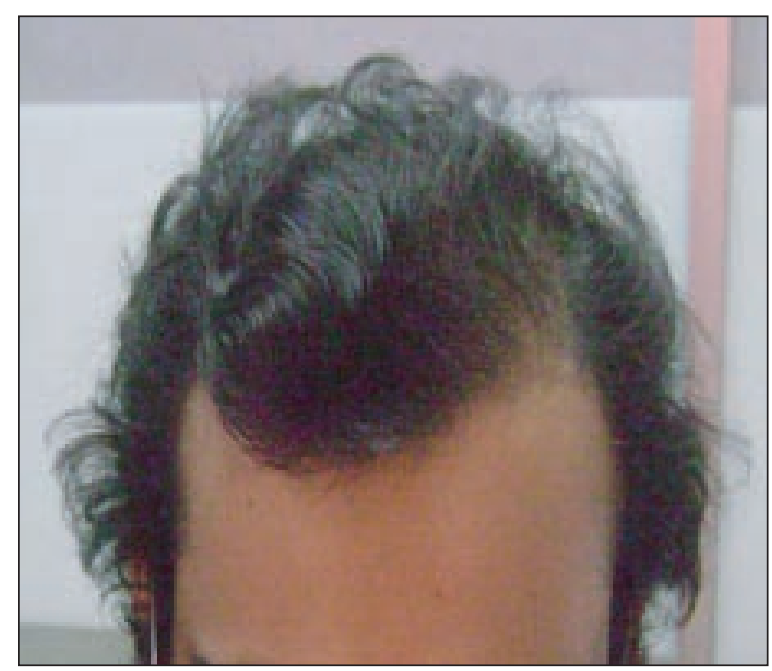

Figure 7: Two years after transplantation 
requires considerable forethought and planning. With better methods of harvesting and implantation, hair transplantation results represent a blend of art and science. Hair transplantation, if done judiciously, is a very rewarding procedure, both for the surgeon and to the patient (fig:6-7).

\section{References}

1. Headington JT. Transverse microscopic anatomy of the human scalp. A basis for a morphometric approach to disorders of the hair follicle. Arch Dermatol. 1984; 120(4): 449-56.

2. Orentreich N. Autografts in alopecias and other selected dermatological conditions. Ann N Y Acad Sci. 20 1959; 83: 463-79.

3. Vogel JE. Hair restoration complications: an approach to the unnatural-appearing hair transplant. Facial Plast Surg. 2008; 24(4): 453-61.

4. Beehner M. Hairline design in hair replacement surgery. Facial Plast Surg. 2008; 24(4): 389-403.

5. Rousso DE, Presti PM. Follicular unit transplantation. Facial Plast Surg. 2008; 24(4): 381-8.

6. Limmer BL. Elliptical donor stereoscopically assisted micrografting as an approach to further refinement in hair transplantation. J Dermatol Surg Oncol. 1994; 20(12): 789-93.

7. Hamilton JB. Patterned loss of hair in man; types and incidence. Ann N Y Acad Sci. 1951; 53(3): 708-28.

8. SeagerD. Binocularstereoscopic dissecting microscopingshould we use them?. Hair Transplant Forum Int. 1996; 6: 2-5.

9. Bernstein RM, Rassman WR, Szaniawski W. Follicular transplantation. Int J Aesthetic Restor Surg. 1995; 3: 119-32. 